\title{
Association of Exosomal miR-34a with Markers of Dyslipidemia and Endothelial Dysfunction in Children and Adolescents with T1DM
}

\author{
(D) Alshaymaa A. Ibrahim ${ }^{1}$, (D) Aliaa A. Wahby ${ }^{1}$, (D) Ingy Ashmawy¹, (D) Rehan M. Saleh², (D) Hend Soliman 3 \\ ${ }^{1}$ National Research Centre, Department of Clinical and Chemical Pathology, Cairo, Egypt \\ ${ }^{2}$ National Research Centre, Department of Community, Cairo, Egypt \\ ${ }^{3}$ Cairo University Faculty of Medicine, New Children Hospital, Cairo, Egypt
}

\begin{abstract}
What is already known on this topic?
Dyslipidemia and endothelial dysfunction are common disorders and major predisposing factors for atherosclerosis and cardiovascular diseases in patients with type 1 diabetes mellitus (T1DM). However, their pathophysiology in children and adolescents with T1 DM is still under evaluated.
\end{abstract}

\section{What this study adds?}

Association of exosomal micro-RNA 34a serum expression with markers of dyslipidemia and endothelial dysfunction was identified in children and adolescents with T1DM, suggesting its role in regulation of lipid metabolism and endothelial function in T1DM.

\section{Abstract}

Objective: Dyslipidemia and endothelial dysfunction are common disorders and major causative factors for atherosclerosis in patients with type 1 diabetes mellitus (T1DM). However, their pathophysiology in young patients with T1DM is still under evaluated. We aimed, for the first time, to assess the expression of exosomal micro-RNA 34a (miR-34a) in serum of children and adolescents with T1DM and correlate this expression with markers of dyslipidemia and endothelial dysfunction.

Methods: The study included 120 T1DM patients and 100 control subjects. Assessment of miR-34a was performed using quantitative real-time polymerase chain reaction. Lipid profile was assessed on an automated analyzer and serum endoglin and intracellular adhesion molecule (ICAM) concentrations were measured using immunometric methods.

Results: Relative expression of miR-34a and serum endoglin and ICAM concentrations were higher in patients than controls $(p=0.001$ ) and in patients with dyslipidemia (42 patients) compared to patients without dyslipidemia (78 patients) $(p=0.01)$. Linear regression analysis revealed a strong independent association between exosomal miR-34a expression and total cholesterol, low-density lipoprotein, serum endoglin and serum ICAM after adjustment for other cofactors. The utility of miR-34a as an indicator for associated dyslipidemia was tested using receiver operator characteristic curve analysis which revealed area under the curve: 0.73 with confidence interval: $0.63-0.83$ and $p=0.001$.

Conclusion: This was the first study to show the altered expression of exosomal miR-34a among children and adolescents with T1DM. Moreover, association of miR-34a with markers of dyslipidemia and endothelial dysfunction was identified, suggesting that it could play a role in regulation of lipid metabolism and endothelial function in T1DM.

Keywords: miR-34a, dyslipidemia, endothelial dysfunction, type 1 diabetes mellitus, endoglin, intracellular adhesion molecule

\section{Introduction}

Type 1 diabetes mellitus (T1DM) is a complex, multifactorial, autoimmune illness and continues to increase in prevalence among children and adolescents (1). T1DM affects about
35 million persons all over the world with annual increase ranging between 3-5\% (2).

Dyslipidemia and endothelial dysfunction are very common metabolic abnormalities in these patients $(3,4)$. Both are major causative factors for atherosclerosis which is a major 
precursor of cardiovascular disease (CVD), the leading cause for morbidity and mortality in T1DM (5). However, the pathophysiology underlying the occurrence of dyslipidemia and endothelial dysfunction in young patients with T1DM remains unclear. It is suggested that the pathogenesis involves an interaction between genetic, environmental and eventually epigenetic factors. Epigenetic factors, including microRNAs, not only represent a key for understanding the complexity of vascular diseases in these patients but also represent a new field of investigation to discover new diagnostic and prognostic markers (6).

MicroRNA (miRNA, miR) is a class of small, noncoding RNA, which play a significant role in regulating gene expression. Therefore, miRNAs could contribute to the pathogenesis of a number of different physiological and pathological processes (7). Exosomes are nanovesicles originating from all cells, whether healthy or diseased, and can be found in all body fluids. The lipid bilayer surrounding exosomes enable exosomal-enclosed miRNAs to be stably expressed in body fluids much more so than free circulating miRNAs. Consequently, recent studies have focused on exosomalenclosed miRNAs as contributing factors in various human diseases (8).

Accumulating data have demonstrated that miR-34a contributes to $\beta$-cell apoptotic pathways, suggesting a role in T1DM (9). Among different miRNA candidates, miR-34a is of special interest regarding lipid metabolism and endothelial function because of its interaction with many genes involved in both pathways (10). Genomic data for exosomal miR$34 \mathrm{a}$ were retrieved from the extracellular vesicles miRNA database $(11,12)$, while the predicted miRNA target genes were analyzed by using DIANA-miRPath v1.1 webserver (13). Accordingly, its role in adipogenesis, atherosclerosis progression, inflammation and CVD development and progression, has been suggested.

The current study aimed, for the first time, to assess the expression of exosomal miR-34a in the serum of children and adolescents with T1DM and to evaluate the association between exosomal miR-34a expression and markers of dyslipidemia, such as total cholesterol (TC), low-density lipoprotein cholesterol (LDL-C), high-density lipoprotein cholesterol (HDL-C) and triglycerides (TG), as well as the serum levels of markers of endothelial dysfunction, including serum endoglin and intracellular adhesion molecule (ICAM).

\section{Methods}

This study is a pilot cross-sectional study. A total of 120 T1DM patients, with disease duration more than five years, were randomly selected from Diabetes, Endocrine and
Metabolism Pediatric Unit, Pediatric Department, Faculty of Medicine, Cairo University, with respect to inclusion and exclusion criteria (see below). Confirmation of T1DM diagnosis was based on criteria of American Diabetes Association (ADA) (14).

- Exclusion criteria in the current study included: any type of diabetes other than T1DM; T1DM with any microvascular complication; hypertension; heart, liver, or renal insufficiency; acute diabetic complications; systemic inflammatory diseases; other endocrine disorders; neoplastic disorders; and family history of dyslipidemic diseases.

- One hundred age and sex matched healthy subjects served as control group and were recruited from the National Research Centre (NRC) during regular check-up.

- The included patients were subsequently categorized into patients with dyslipidemia (42 patients) and patients without dyslipidemia (78 patients). According to the ADA, dyslipidemia was defined by the presence of one or more of the following criteria: TC $\geq 200 \mathrm{mg} / \mathrm{dL}$, LDL-C $\geq 130 \mathrm{mg} / \mathrm{dL}$, HDL-C $\leq 35 \mathrm{mg} / \mathrm{dL}$, and TG $\geq 150 \mathrm{mg} / \mathrm{dL}$ (15).

- Informed consent was obtained from each participant. This study was approved by the Ethics Committee of the NRC, under approval number $16 / 285$, in accordance with the Declaration of Helsinki 2015.

All participants were subjected to full history taking and full clinical examination.

\section{Biochemical Analysis}

- Venous blood samples were collected from all subjects after 12 hours of overnight fast. Serum was separated and parameters of dyslipidemia including, TC, TG, HDL and LDL levels were quantified using Erba Mannheim XL300 Chemistry Analyzer (ERBA Diagnostics Mannheim, Medical EXPO, India).

- Markers of endothelial dysfunction including serum endoglin and ICAM concentrations were assessed using enzyme linked immunosorbent assay sandwich technique (Quantikine, R\&D Systems, Minneapolis, USA).

Assessment of exosomal miR-34a was done by quantitative reverse transcriptase real-time polymerase chain reaction (qRT PCR) technique:

Exosomes were isolated from eight hundred microliters of serum according to exoRNeasy Serum/Plasma midi kit (Qiagen, Hilden, Germany). The characteristics of isolated exosomes were confirmed by transmission electron microscopy (16). Isolation, purification and elution of 
exosomal RNA was done according to exoRNeasy Serum/ Plasma midi kit's protocol and 3.5 microns of synthetic spike in control Cel-miR-39 was added to each sample as the internal control. Concentration of isolated RNA was assessed using a NanoDrop 2000c spectrophotometer (ThermoFisher Scientific, Waltham, Massachusetts, USA). Complementary DNA (CDNA) was synthesized using a miScript reverse transcription kit (Qiagen, Hilden, Germany) and then all specimens were stored at -80 ${ }^{\circ} \mathrm{C}$. Quantitative PCR was run on Applied technologies, Stratagene Mx3000P using miScript SYBR green PCR kit (Qiagen, Hilden, Germany) for detection of miR-34a (ID: MS00003318). The relative expression of miRNA was described as fold change using the calculated formula; $2-\Delta \Delta C T$. Methods have been described in detail elsewhere (8).

\section{Statistical Analysis}

Patients' clinical and laboratory quantitative and qualitative data were presented as mean \pm standard deviation and frequencies respectively, while the levels of relative miR34 expression were presented as median [interquartile range (IQR)]. Non-parametric tests were used to evaluate expression difference of miR-34a between patients and healthy controls and between patients' groups. To assess the relationships between exosomal miR-34a and different patients' parameters, Pearson's correlation and linear regression were performed. The utility of miR-34a as an indicator for associated dyslipidemia among patients was tested using receiver operating curve (ROC) technique and area under the curve (AUC) was calculated. All tests were two-sided and a $p<0.05$ was considered statistically significant.

\section{Results}

The clinical, demographic and routine laboratory test results for patients and healthy controls are presented in Table 1 . Both patients and controls were matched for age, gender and body mass index (BMI). Glycated hemoglobin (HbA1c), TC, TGs and LDL were significantly increased in patients compared to controls, while HDL showed no significant difference between the two groups.

Regarding miRNA expression, miR-34a showed significant higher expression among T1DM patients (median: 22.6, IQR: 4.2-111.7 fold change) than healthy controls (median: 6, IQR: 0.1-12 fold change) ( $p=0.001$ ) (Figure 1). Frequency of patients with miR-34a overexpression, defined as expression more than two-fold change, among T1DM patients was $90 \%$. Association of miR-34a expression and T1DM was confirmed using logistic regression analysis after adjustment for age, gender and BMI $(p=0.01)$.

Comparison between patients regarding associated dyslipidemia showed that the relative expression of exosomal miR-34a was higher in patients with dyslipidemia (median: 78; IQR: 18.1-2388 fold change) compared to patients without dyslipidemia (median: 4.8; IQR: 3.734.2 fold change) $(p=0.001)$ (Figure 1$)$. There was no significant difference between patients with dyslipidemia and those without dyslipidemia regarding age, gender, BMI and HbA1c. Disease duration, serum TC, TGs and LDL-C were significantly higher in patients with dyslipidemia compared to patients without dyslipidemia, while HDL showed no significant difference between the two groups (Table 2). The most common disorder of dyslipidemia was hypercholesterolemia (95\%). Prevalence of high TG, low HDL-C and high LDL-C was $9.5 \%, 12 \%$ and $45 \%$ respectively.

\begin{tabular}{llll}
\hline \multicolumn{4}{l}{ Table 1. Clinical, demographic and biochemical laboratory data of patients and controls } \\
\hline Characteristic & Controls (number = 100) & T1DM (number = 120) & p value \\
\hline Age (years) & $12.1 \pm 2.8$ & $13.5 \pm 3.2$ & 0.6 \\
Gender (male/female) & $41 / 59$ & $54 / 66$ & 0.5 \\
BMI (kg/m²) & $18.1 \pm 3.1$ & $18.9 \pm 2.3$ & 0.7 \\
Diabetes duration (years) & & $7.6 \pm 1.9$ & - \\
HbA1c (\%) & $4 \pm 0.6$ & $8 \pm 1.2$ & 0.001 \\
Total cholesterol (mmol/L) & $127 \pm 25$ & $181 \pm 42$ & 0.001 \\
Triglycerides (mg/dL) & $75 \pm 35$ & $88 \pm 30.7$ & 0.005 \\
HDL-C (mg/dL) & $52 \pm 4.4$ & $53 \pm 8.8$ & 0.2 \\
LDL-C (mg/dL) & $80 \pm 19.1$ & $106 \pm 33$ & 0.001 \\
Serum endoglin (ng/mL) & $28.5(16.8-43)$ & $31(19-75)$ & 0.01 \\
ICAM (ng/mL) & $231(129-321)$ & $293(240-693)$ & 0.001 \\
\hline
\end{tabular}

T1DM: type 1 diabetes mellitus, BMI: body mass index, HbA1c: glycated hemoglobin, HDL-C: high density lipoprotein-cholesterol, LDL-C: low density lipoprotein-cholesterol, ICAM: intracellular adhesion molecule 
Levels of serum endoglin and serum ICAM were significantly higher in patients with T1DM than healthy subjects ( $p=0.01$ and $p=0.001$, respectively) (Table 1 ) and higher in T1DM patients with dyslipidemia compared to patients without dyslipidemia ( $p=0.01$ ) (Table 2). Serum endoglin and serum

\section{A}

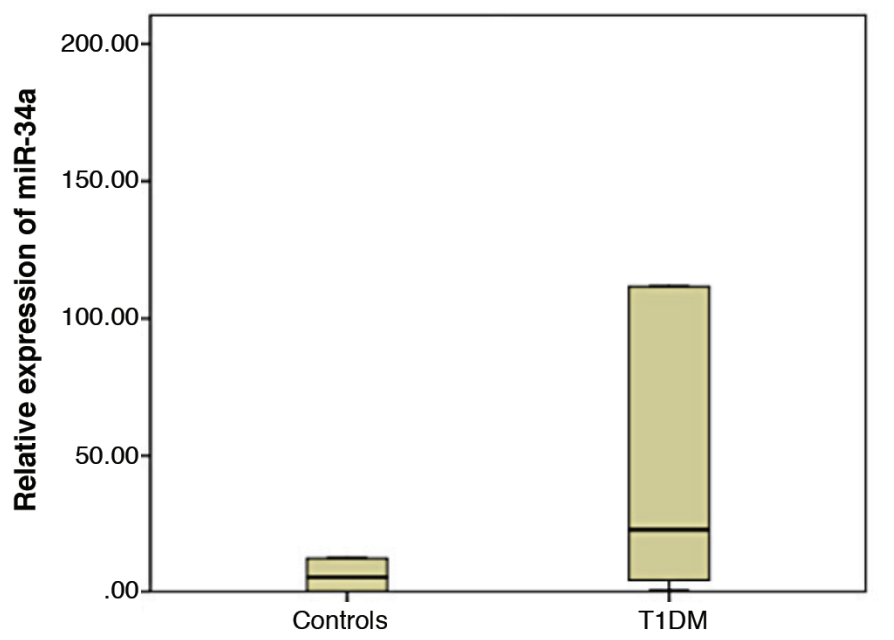

ICAM showed no significant correlation with age, BMI, disease duration, glycated hemoglobin and parameters of the lipid profile (Table 3).

Pearson's correlation revealed positive correlation between miR-34a and both serum endoglin and serum

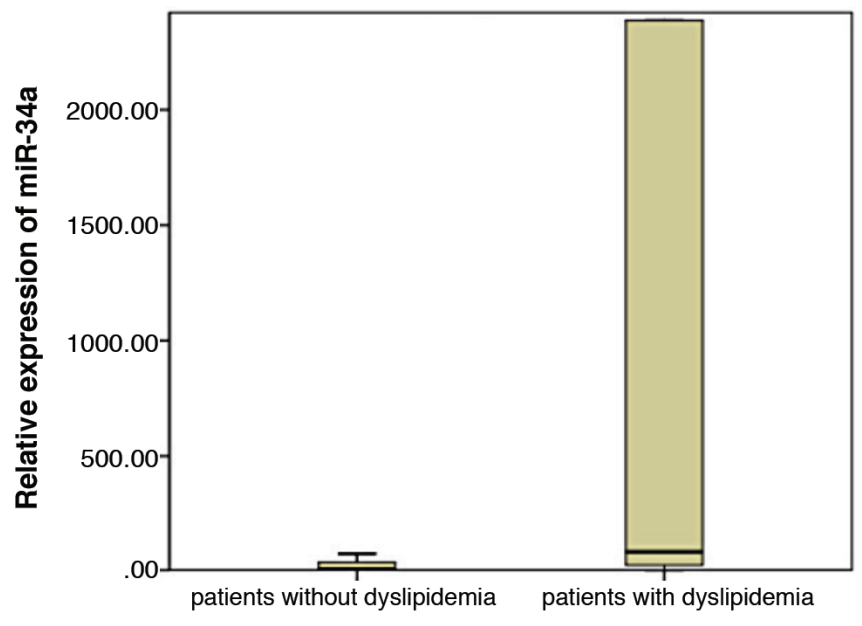

Figure 1. Expression of exosomal micro-RNA 34a (miR-34a) among studied groups. A) Shows significant higher expression of miR-34a in patients with type 1 diabetes mellitus [median: 22.6, interquartile range (IQR): 4.2-111.7] compared to healthy controls (median: 6, IQR: 0.1-12). B) Shows increased expression of exosomal miR-34a in patients with dyslipidemia (median: 78, IQR: 18.1-2388) compared to patients without dyslipidemia (median: 4.8, IQR: 3.7-34.2)

miR-34a: micro-RNA 34a, T1DM: type 1 diabetes mellitus

Table 2. Comparison between patients with dyslipidemia and patients without dyslipidemia

\begin{tabular}{llll}
\hline Parameter & $\begin{array}{l}\text { Patients without } \\
\text { dyslipidemia } \\
\text { (78 patients) }\end{array}$ & $\begin{array}{l}\text { Patients with dyslipidemia } \\
\text { (42 patients) }\end{array}$ & p value \\
\hline Age (years) & $13.6 \pm 3.2$ & $13.2 \pm 3.4$ & 0.5 \\
Gender (male/female) & $32 / 46$ & $22 / 20$ & 0.2 \\
BMI $\left(\mathrm{kg} / \mathrm{m}^{2}\right)$ & $18.8 \pm 3.1$ & $17.9 \pm 3.6$ & 0.9 \\
Disease duration (years) & $7.2 \pm 1.6$ & $8.3 \pm 2.2$ & 0.03 \\
HbA1c (\%) & $7.9 \pm 1.1$ & $8.3 \pm 1.2$ & 0.1 \\
Total cholesterol (mmol/L) & $157 \pm 23.2$ & $227 \pm 20.3$ & 0.001 \\
Triglycerides $(\mathrm{mg} / \mathrm{dL})$ & $80.3 \pm 43$ & $103 \pm 25.7$ & 0.001 \\
HDL-C $(\mathrm{mg} / \mathrm{dL})$ & $52.6 \pm 8.3$ & $54 \pm 9.7$ & 0.2 \\
LDL-C (mg/dL) & $87.8 \pm 21.4$ & $139.5 \pm 25.3$ & 0.001 \\
Frequency of disorders & & & \\
Hypercholesterolemia $(>200 \mathrm{mmol} / \mathrm{L})$ & - & $40 / 42$ & - \\
Hypertriglyceridemia $(>150 \mathrm{mg} / \mathrm{dL})$ & - & $4 / 42$ & \\
Decreased HDL-C $(<35 \mathrm{mg} / \mathrm{dL})$ & - & $5 / 42$ & 0.02 \\
Increased LDL-C $(>130 \mathrm{mg} / \mathrm{dL})$ & - & $48 / 42$ & 0.005 \\
Serum endoglin $(\mathrm{ng} / \mathrm{mL})$ & $29(18-57)$ & $435(346-657)$ & \\
ICAM (ng/mL) & $342(234-509)$ & & \\
\hline
\end{tabular}

BMI: body mass index, HbA1c: glycated hemoglobin, HDL-C: high density lipoprotein-cholesterol, LDL-C: low density lipoprotein-cholesterol, ICAM: intracellular adhesion molecule 
ICAM concentration, but failed to demonstrate significant association between miR-34a with parameters of lipid profile (Table 4). Linear regression analysis was used to confirm the association of exosomal expression of miR$34 \mathrm{a}$ with parameters of lipid metabolism and endothelial dysfunction in patients with T1DM after adjustment for age, gender, BMI and disease duration. This analysis revealed a strong independent association between exosomal miR-34a with TC, serum endoglin and serum ICAM (Table 4). The utility of miR-34a as indicator for associated dyslipidemia was tested using ROC curve which revealed AUC: 0.73 with confidence intervals: 0.63-0.83 ( $p=0.001)$ (Figure 2).

To validate and strengthen our results, the analysis of miRNAs that target different genetic pathways involved in lipid metabolism and endothelial function was done using https://ccb-web.cs.uni-saarland.de/mirtargetlink/index.php. This analysis retrieved miR-34a as one of the three miRNAs that can target both the hepatocyte nuclear factor 4 (HNF4) and sirtuin 1 (SIRT1) genes that have been identified as playing major roles in lipid metabolism (Figure 3); In addition miR-34a was the only miRNA that targeted the three major genes, vascular endothelial growth factor (VEGF), SIRT1 and p53, involved in endothelial function (Figure 4) (17).

\section{Discussion}

Pathogenesis of associated endothelial dysfunction and dyslipidemia in children and adolescents with T1DM is still under-evaluated. This is the first study to evaluate exosomal miR-34a expression in children and adolescents with T1DM and to correlate this expression with markers of dyslipidemia and endothelial dysfunction in the studied patients.

The role of miR-34a in the development of diabetes is under the spotlight. Accumulating data indicate that miR-34a plays significant roles in glucose sensing, insulin secretion, and increasing sensitivity of $\beta$ cells to cytokine-induced apoptosis $(18,19,20)$. In the current study, expression of miR-34a was increased in patients with T1DM compared to healthy subjects, suggesting its role in the pathogenesis of T1DM. This is in agreement with other studies that demonstrated overexpression of miR-34a in T1DM $(21,22)$, especially in recentonset T1DM, compared to high-risk and healthy children (23).

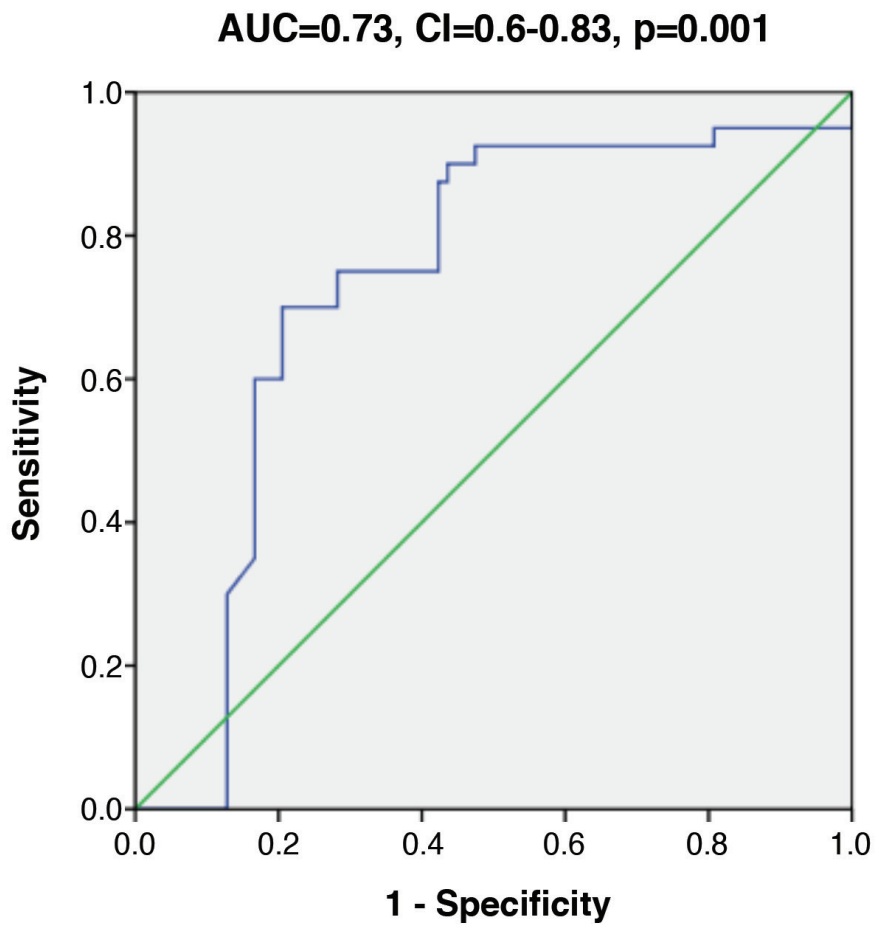

Figure 2. Receiver operating curve (ROC) curve of exosomal micro-RNA 34a (miR-34a) in associated dyslipidemia in type 1 diabetes mellitus. ROC curve showed the utility of miR-34a as an indicator of associated dyslipidemia. Area under the curve: 0.73 with confidence intervals: 0.63-0.83 $(p=0.001)$

AUC: area under the curve, CI: confidence interval

Table 3. Pearson's correlation of serum endoglin and serum intracellular adhesion molecule with different parameters in type 1 diabetes mellitus patients

\begin{tabular}{lllll}
\hline Parameters & \multicolumn{2}{l}{ Serum endoglin } & & \multicolumn{2}{l}{ Serum ICAM } \\
\cline { 2 - 5 } & $\mathrm{r}$ & $\mathrm{p}$ value & $\mathrm{r}$ & $\mathrm{p}$ value \\
\hline Age (years) & -0.1 & 0.1 & -0.1 & 0.2 \\
BMI (kg/m²) & 0.3 & 0.09 & 0.09 & 0.1 \\
Disease duration (years) & 0.07 & 0.4 & -0.2 & 0.6 \\
HbA1c (\%) & 0.13 & 0.52 & -0.002 & 0.9 \\
Total cholesterol (mmol/L) & 0.09 & 0.1 & -0.03 & 0.73 \\
Triglycerides (mg/dL) & -0.06 & 0.6 & 0.03 & 0.75 \\
HDL-C (mg/dL) & 0.01 & 0.8 & 0.04 & 0.51 \\
LDL-C (mg/dL) & 0.08 & 0.1 & 0.2 & 0.2 \\
\hline I: Pears/scorr
\end{tabular}

r: Pearson's correlation coefficient, BMI: body mass index, HbA1 c: glycated hemoglobin, HDL-C: high density lipoprotein-cholesterol, LDL-C: low density lipoprotein-cholesterol, ICAM: intracellular adhesion molecule 
Dyslipidemia is a metabolic disorder commonly associated with T1DM, increasing the risk of CVD (5). In our study, the prevalence of dyslipidemia among patients with T1DM was $35 \%$. El-Bakry et al (24) reported that $64 \%$ of Egyptian children and adolescents with T1DM showed association with dyslipidemia. In addition, the prevalence of dyslipidemia in children and adolescents with T1DM is

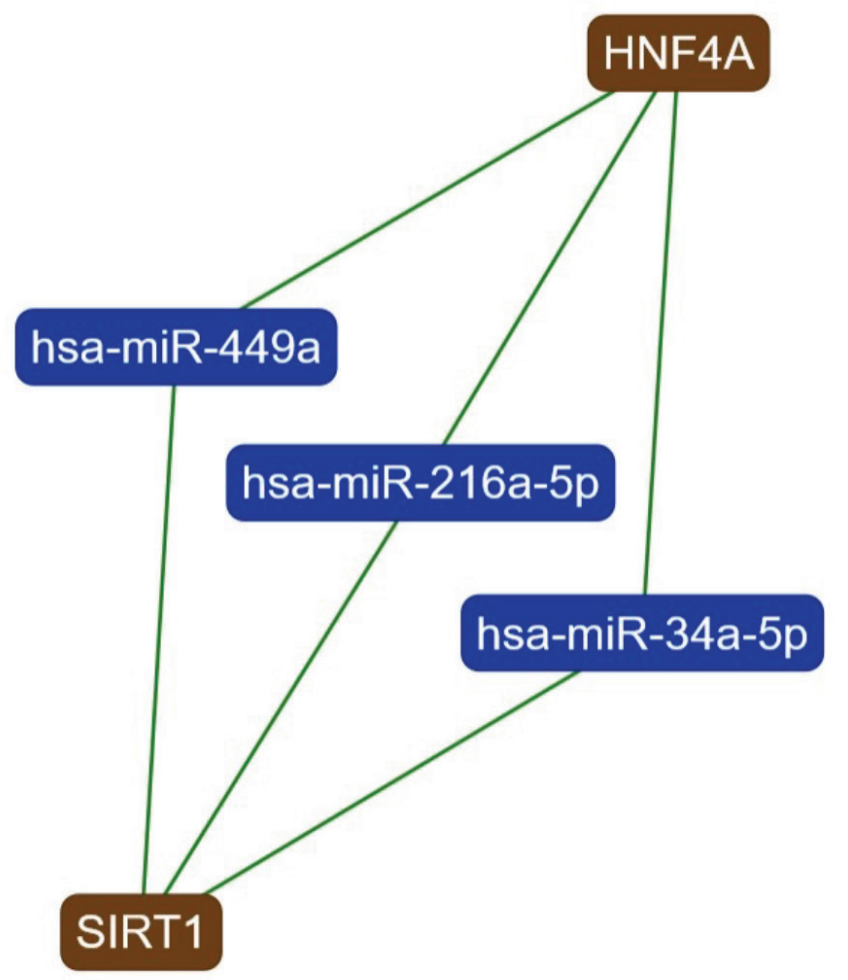

Figure 3. Interaction network of genes targeted by micro-RNA 34a (miR-34a) and playing a significant role in lipid metabolism. This analysis was done using (miRTargetLinkdatabase) (https://ccb-web.cs.uni-saarland.del mirtargetlink/index.php) and retrieved that miR-34a is one of the three miRNAs that can target both hepatocyte nuclear factor 4 (HNF4) and the sirtuin 1 (SIRT1) genes (green line indicates a strong interaction) reported to vary from $29 \%$ to $66 \%$ in cross sectional studies from different countries $(25,26,27)$. The most common types of dyslipidemia among adolescents with T1DM also varies between hypercholesterolemia $(24,28)$, high triglyceridemia $(29,30)$ and high LDL-C $(31,32)$. Differences in sample size, inclusion and exclusion criteria, degree of glycemic control and ethnicity might be the cause of this wide range of prevalences and difference in frequencies of dyslipidemia types in the previous studies.

MiR-34a is known to regulate TC and hepatic lipid metabolism through targeting and inhibition of expression of the NAD + dependent lysine deacetylase SIRT1, the antiatherogenic mediator that regulates lipid metabolism and endothelial function (33). MiR-34a also down regulates HNF4, a gene which modulates the expression of other genes involved in lipid and glucose metabolism (34). In agreement with the previous data, we have demonstrated for the first time increased miR-34a expression in patients with dyslipidemia compared to those without dyslipidemia. Moreover, miR$34 \mathrm{a}$ overexpression was described in different diseases associated with dyslipidemia, particularly non-alcoholic fatty liver disease $(33,35)$ and coronary artery disease $(36)$ and this expression was correlated with disease severity. Linear regression analysis for our results revealed positive association between miR-34a and TC and LDL-C, while no correlation or association was detected between the miRNA and TG. Interestingly, Shen et al (20) reported positive correlation of miR-34a expression with LDL-C and negative correlation with TG in patients with T2DM. However, Xu et al (34) suggested an explanation when they showed that miR34a regulates hepatic TGs via regulation of HNF4 expression and its overexpression leads to accumulation of TGs in the liver and subsequently decreasing serum TGs.

Endothelial dysfunction precedes and promotes vascular inflammation and therefore atherosclerosis in T1DM and may be considered as an independent predictor of associated CVD (3). Endoglin (CD105) is a membrane

Table 4. Pearson's correlation and linear regression analysis of micro-RNA 34a with different parameters of dyslipidemia and endothelial dysfunction

\begin{tabular}{lllll}
\hline Parameters & \multicolumn{2}{l}{ Pearson's correlation } & & \multicolumn{2}{l}{ Regression analysis } \\
\cline { 2 - 5 } & $\mathrm{r}$ & $\mathrm{p}$ value & $\beta$ & 0.001 \\
\hline Total cholesterol (mmol/L) & 0.13 & 0.03 & 0.56 & 0.9 \\
Triglycerides (mg/dL) & 0.006 & 0.3 & 0.1 & 0.03 \\
HDL-C (mg/dL) & 0.12 & 0.07 & 0.3 & 0.01 \\
LDL-C (mg/dL) & 0.1 & 0.03 & 0.65 & 0.001 \\
Serum endoglin $(\mathrm{ng} / \mathrm{mL})$ & 0.6 & 0.001 & 0.51 & 0.001 \\
Serum ICAM (ng/mL) & 0.45 & 0.001 & & 0.01 \\
\hline
\end{tabular}

r: Pearson's correlation coefficient, $\beta$ : standardized regression coefficient, HDL-C: high density lipoprotein-cholesterol, LDL-C: low density lipoproteincholesterol, ICAM: intracellular adhesion molecule 


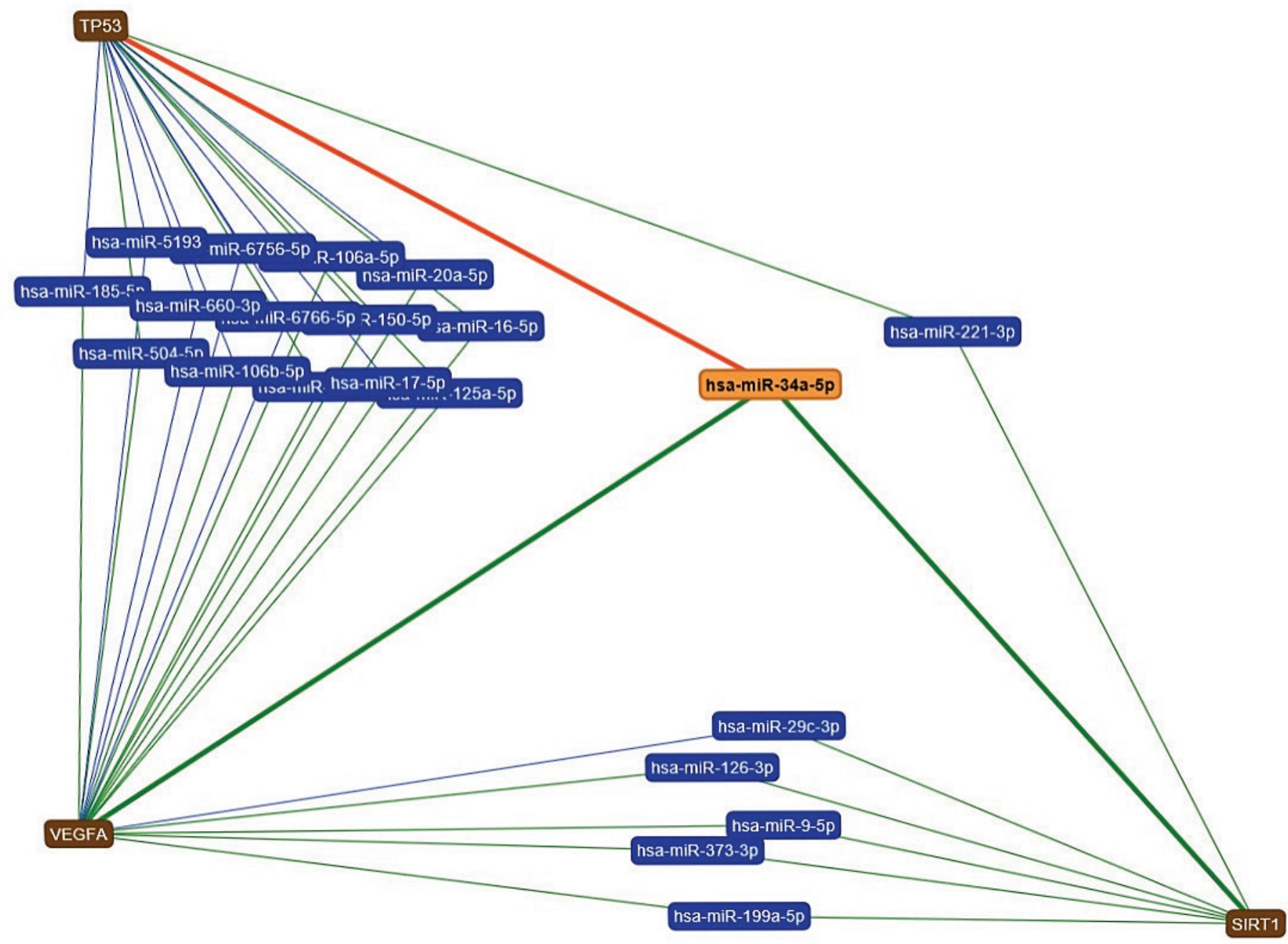

Figure 4. Interaction network of genes targeted by micro-RNA 34a (miR-34a) and playing a significant role in endothelial function. This analysis was done using (miRTargetLinkdatabase) (https://ccb-web.cs.uni-saarland.de/mirtargetlink/index.php) and retrieved that miR-34a is the only miRNA that targets the three major genes, vascular endothelial growth factor, sirtuin 1 (SIRT1) and p53, involved in endothelial function (green line indicates a strong interaction and red line indicates a weak interaction)

glycoprotein located on the surface of vascular endothelial cells. Endoglin, acts as a receptor for transforming growth factor-beta (TGF- $\beta$ ) which is an important mediator for angiogenesis and responsible for keeping the vascular endothelium healthy (37). ICAM is a surface adhesion molecule, also expressed on vascular endothelial cells and also immune cells, which facilitates cell-to-cell interaction and recruitment of leucocytes to endothelium during inflammation (38). Shedding of endoglin and ICAM1 receptors into the systemic circulation during endothelial injury renders them potential circulatory markers of endothelial dysfunction (37). In this study, levels of serum endoglin and serum ICAM were highly elevated in patients with T1DM compared to healthy controls. In agreement with our results, there have been previous reports of elevated levels of serum endoglin and serum ICAM in patients with T1DM $(38,39,40)$. Another interesting finding was the elevation of serum endoglin and serum ICAM in patients with dyslipidemia compared to patients without dyslipidemia, in agreement to El-Kassas et al (39), who reported significant positive correlations of endoglin with TC, TG and LDL-C in children with T1DM. In addition, serum endoglin and serum ICAM showed positive correlations with exosomal miR-34a expression in our study. This data was consistent with other reports, stating that miR-34a is one of the most important endothelial miRNAs which plays a significant role in maintaining endothelial cell function by targeting many 
genes including p53, VEGF and SIRT1 (41). Moreover, TGF- $\beta$ increases endoglin and can upregulate miR-34a, which subsequently promotes vascular inflammation by upregulating vascular cell adhesion molecule- 1 and ICAM $(10,42)$. Interestingly, it was reported that miR-34a deletion in the endothelium preserve endothelial functions in diabetic mice indicating the responsibility of miR-34a for diabetic vascular dysfunction (10).

\section{Study Limitations}

The partial small sample size and the cross-sectional research design may be considered as limitations for this study.

\section{Conclusion}

To the best of our knowledge, this is the first study to report the altered expression of exosomal miR-34a among children and adolescents withT1DM. Moreover, association of miR-34a with markers of dyslipidemia and endothelial dysfunction was demonstrated, suggesting a role for miR34 in the epigenetic regulation of lipid metabolism and endothelial function in T1DM children and adolescents. More longitudinal studies with larger sample sizes and prospective design are warranted to investigate this association further. Future studies are recommended to identify the possible use of miR-34a as a therapeutic target in patients with T1DM and CVD.

\section{Ethics}

Ethics Committee Approval: This study was approved by the Ethics Committee of the NRC, under approval number 16/285, in accordance with the Declaration of Helsinki 2015.

Informed Consent: Informed consent was obtained from each participant.

Peer-review: Externally peer-reviewed.

\section{Authorship Contributions}

Surgical and Medical Practices: Alshaymaa A. Ibrahim, Aliaa A. Wahby, Ingy Ashmawy, Rehan M. Saleh, Hend Soliman, Concept: Alshaymaa A. Ibrahim, Aliaa A. Wahby, Ingy Ashmawy, Rehan M. Saleh, Hend Soliman, Design: Alshaymaa A. Ibrahim, Aliaa A. Wahby, Ingy Ashmawy, Rehan M. Saleh, Hend Soliman, Data Collection or Processing: Alshaymaa A. Ibrahim, Aliaa A. Wahby, Ingy Ashmawy, Rehan M. Saleh, Hend Soliman, Analysis or Interpretation: Alshaymaa A. Ibrahim, Aliaa A. Wahby, Ingy Ashmawy, Rehan M. Saleh, Hend Soliman, Literature Search: Alshaymaa A. Ibrahim, Aliaa A. Wahby, Ingy Ashmawy, Rehan M. Saleh, Hend
Soliman, Writing: Alshaymaa A. Ibrahim, Aliaa A. Wahby, Ingy Ashmawy, Rehan M. Saleh, Hend Soliman.

Financial Disclosure: This work was supported by National Research Centre, Centre of Excellence.

\section{References}

1. Ahmed AE, Sakhr HM, Hassan MH, El-Amir MI, Ameen HH. Vitamin D receptor rs7975232, rs731236 and rs1544410 single nucleotide polymorphisms, and 25-hydroxyvitamin D levels in Egyptian children with type 1 diabetes mellitus: effect of vitamin D co-therapy. Diabetes Metab Syndr Obes 2019;12:703-716.

2. Mostofizadeh N, Hashemipour M, Roostazadeh M, Hashemi-Dehkordi E, Shahsanai A, Reisi M. The impact of poor glycemic control on lipid profile variables in children with type 1 diabetes mellitus. J Educ Health Promot 201 9;8:6

3. Ladeia AM, Sampaio RR, Hita MC, Adan LF. Prognostic value of endothelial dysfunction in type 1 diabetes mellitus. World J Diabetes 2014;5:601-605

4. Ahmed TA, Ahmed YA, Arafa AI, Salah RA. Detection of occult right ventricular dysfunction in young Egyptians with type 1 diabetes mellitus by two-dimensional speckle tracking echocardiography. Indian Heart J 2018;70:665-671. Epub 2018 Jul 6

5. Kim SH, Jung IA, Jeon YJ, Kyoung Cho W, Soon Cho K, Park SH, Jung $\mathrm{MH}$, Suh BK. Serum lipid profiles and glycemic control in adolescents and young adults with type 1 diabetes mellitus. Ann Pediatr Endocrinol Metab 2014;19:191-196. Epub 2014 Dec 31

6. Palacio Rojas M, Prieto C, Bermúdez V, Garicano C, Nava TN, Martínez MS, Salazar J, Rojas E, Pérez A, Vicuña PM, Martínez NG, Parra SM, Hoedebecke K, D’Addosio R, Cano C, Rojas J. Dyslipidemia: Genetics, lipoprotein lipase and HindIII polymorphism. F1000Res 2017;6:2073.

7. Ibrahim AA, Ramadan A, Wahby AA, Hassan M, Soliman HM, Abdel Hamid TA. Micro-RNA 196a2 expression and miR-196a2 (rs11614913) polymorphism in T1DM: a pilot study. J Pediatr Endocrinol Metab 2019;32:1171-1179.

8. Ibrahim A, Soliman H, El-Lebedy D, Hassan M, Helmy N, Hamid T, Abdelhamid N. Expression of exosomal miR-21 and miR-29 in serum of children and adolescents with T1DM and persistent microalbuminuria. Gene Rep 2019;16:100461

9. Acke MB, Novotny GW, Christensen DP, Grunnet LG, Mandrup-Poulsen T. Altering $\beta$-cell number through stable alteration of miR-2 1 and miR34a expression. Islets 2014;6:e27754.

10. Li Q, Kim YR, Vikram A, Kumar S, Kassan M, Gabani M, Ki Lee S, Jacobs JS, Irani K. P66Shc-Induced MicroRNA-34a Causes Diabetic Endothelial Dysfunction by Downregulating Sirtuin 1. Arterioscler Thromb Vasc Biol 2016;36:2394-2403. Epub 2016 Oct 27

11. EVmiRNA: The Extracellular Vesicles miRNA Database. Last accessed date: 2 June 2020. Available at: http://bioinfo.life.hust.edu.cn/ EVmiRNA\#!/

12. Liu T, Zhang Q, Zhang J, Li C, Miao YR, Lei Q, Li Q, Guo AY. EVmiRnA: a database of miRNA profiling in extracellular vesicles. Nucleic Acids Res 2019;47:D89-D93.

13. miRPathDB 2.0 Last accessed date: 5 May 2020. Available at: https:// mpd.bioinf.uni-sb.de/overview.html

14. American Diabetes Association. Classification and Diagnosis of Diabetes. Diabetes Care 2017;40(Suppl 1):S11-S24.

15. American Diabetes Association. Management of dyslipidemia in children and adolescents with diabetes. Diabetes Care 2003;26:21942197. 
16. Kobayashi M, Sawada K, Nakamura K, Yoshimura A, Miyamoto M, Shimizu A, Ishida K, Nakatsuka E, Kodama M, Hashimoto K, Mabuchi S, Kimura T. Exosomal miR-1290 is a potential biomarker of highgrade serous ovarian carcinoma and can discriminate patients from those with malignancies of other histological types. J Ovarian Res 2018;11:81.

17. miRTargetLink Human. Last Accessed date: 2 June 2020. Available from: https://ccb-web.cs.uni-saarland.de/mirtargetlink/

18. Kong L, Zhu J, Han W, Jiang X, Xu M, Zhao Y, Dong Q, Pang Z, Guan $\mathrm{Q}$, Gao L, Zhao J, Zhao L. Significance of serum microRNAs in prediabetes and newly diagnosed type 2 diabetes: a clinical study. Acta Diabetol 2011;48:61-69. Epub 2010 Sep 21

19. Bache MB, Novotny GW, Christensen DP, Grunnet LG, Mandrup-Poulsen T. Altering $\beta$-cell number through stable alteration of miR-21 and miR34a expression. Islets 2014;6:e27754.

20. Shen Y, Xu H, Pan X, Wu W, Wang H, Yan L, Zhang M, Liu X, Xia S, Shao Q. miR-34a and miR-125b are upregulated in peripheral blood mononuclear cells from patients with type 2 diabetes mellitus. Exp Ther Med 2017;14:5589-5596. Epub 2017 Oct 3

21. par Spagnuolo I, Sebastiani G, Patti A, Grieco FA, Ferretti E, Tiberti C, Dotta F. Specific microRNAs expression fingerprint in serum of type 1 diabetic patient. Diabetologia 2012;55:S48.

22. Seyhan AA, Nunez Lopez YO, Xie H, Yi F, Mathews C, Pasarica M, Pratley RE. Pancreas-enriched miRNAs are altered in the circulation of subjects with diabetes: a pilot cross-sectional study. Sci Rep 2016;6:31479.

23. Åkerman L, Casas R, Ludvigsson J, Tavira B, Skoglund C. Serum miRNA levels are related to glucose homeostasis and islet autoantibodies in children with high risk for type 1 diabetes. PLoS ONe 2018;13:e0191067.

24. El-Bakry MM, Mansour AI, Ahmed ME, Sptan HE. Dyslipidemia in Egyptian children and adolescents with type 1 diabetes mellitus. Benha Med J 2017;34:104-107.

25. Homma TK, Endo CM, Saruhashi T, Mori AP, Noronha RM, Monte O, Procópio Calliari ET. Dyslipidemia in young patients with type 1 diabetes mellitus. Arch Endocrinol Metab 2015;59:215-219.

26. Bulut T, Demirel F, Metin A. The prevalence of dyslipidemia and associated factors in children and adolescents with type 1 diabetes. J Pediatr Endocrinol Metab 2017;30:181-187.

27. Zabeen B, Balsa AM, Islam N, Parveen M, Nahar J, Azad K. Lipid profile in relation to glycemic control in type 1 diabetes children and adolescents in Bangladesh. Indian J Endocrinol Metab 2018;22:89-92.

28. Schwab KO, Doerfer J, Hecker W, Grulich-Henn J, Wiemann D, Kordonouri O, Beyer P, Holl RW, DPV Initiative of the German Working Group for Pediatric Diabetology. Spectrum and prevalence of atherogenic risk factors in 27,358 children, adolescents, and young adults with type 1 diabetes: cross-sectional data from the German diabetes documentation and quality management system (DPV). Diabetes Care 2006;29:218-225.

29. Herman WH, Aubert RE, Engelgau MM, Thompson TJ, Ali MA, Sous ES, Hegazy M, Badran A, Kenny SJ, Gunter EW, Malarcher AM, Brechner
RJ, Wetterhall SF, DeStefano F, Smith PJ, Habib M, abd el Shakour S, Ibrahim AS, el Behairy EM. Diabetes mellitus in Egypt: Glycaemic control and microvascular and neuropathic complications. Diabet Med 1998;15:1045-1051.

30. Kantoosh MM, Naiem AM, El-Sayad M, Nashat M. Dyslipidemia and lipid peroxidation in type 1 diabetic children with good glycemic control: Response to antioxidant therapy. Alex J Pediatr 2002;16:357364.

31. al-Naama LM, Kadhim M, al-Aboud MS. Lipid profile in children with insulin dependent diabetes mellitus. J Pak Med Assoc 2002;52:29-34.

32. Rahma S, Rashid JA, Farage AH. The significance of lipid abnormalities in children with insulin dependent diabetes mellitus. Iraqi Postgrad Med J 2006;5:289-294.

33. Hendy OM, Rabie H, El Fouly A, Abdel-Samiee M, Abdelmotelb N, Elshormilisy AA, Allam M, Ali ST, Bahaa El-Deen NM, Abdelsattar S, Mohamed SM. The Circulating Micro-RNAs (-122, -34a and -99a) as Predictive Biomarkers for Non-Alcoholic Fatty Liver Diseases. Diabetes Metab Syndr Obes 2019;12:2715-2723. Epub 2019 Nov 20

34. Xu Y, Zalzala M, Xu J, Li Y, Yin L, Zhang Y. A metabolic stress-inducible miR-34a-HNF4 $\alpha$ pathway regulates lipid and lipoprotein metabolism. Nat Commun 2015;6:7466.

35. Salvoza NC, Klinzing DC, Gopez-Cervantes J, Baclig MO. Association of Circulating Serum miR-34a and miR-122 with Dyslipidemia among Patients with Non-Alcoholic Fatty Liver Disease. PLoS One 2016;11:e0153497.

36. Tabuchi T, Satoh M, Itoh T, Nakamura M. MicroRNA-34a regulates the longevity-associated protein SIRT1 in coronary artery disease: effect of statins on SIRT1 and microRNA-34a expression. Clin Sci (Lond) 2012;123:161-171.

37. Jang YS, Choi IH. Contrasting roles of different endoglin forms in atherosclerosis. Immune Netw 2014;14:237-240.

38. Głowinska B, Urban M, Peczynska J, Szczepanska-Kostro J. Selected adhesion molecules: sICAM-1 and SVCAM-1 as markers of endothelial dysfunction in diabetic children and adolescence. Pol Merkur Lekarski 2003;14:205-209.

39. El-Kassas G, Wakeel ME, Helal R, Amer AF, Elbatal WH, Amer MF, ElGhaffar NA. Endoglin: a novel predictor of vascular complications in type 1 diabetic children and adolescents. JASMR 2017;12:73-78.

40. Rostampour N, Fekri K, Hashemi-Dehkordi E, Obodiat M. Association between vascular endothelial markers and carotid intima-media thickness in children and adolescents with type 1 diabetes mellitus. J Clin Diagn Res 2017;11:SC01-SC05. Epub 2017 Sep 1

41. Shah D, Das P, Alam MA, Mahajan N, Romero F, Shahid M, Singh H, Bhandari V. MicroRNA-34a Promotes Endothelial Dysfunction and Mitochondrial-mediated Apoptosis in Murine Models of Acute Lung Injury. Am J Respir Cell Mol Biol 2019;60:465-477.

42. Takano M, Nekomoto C, Kawami M, Yumoto R. Role of miR-34a in TGF- $\beta 1$ - and drug-induced epithelial-mesenchymal transition in alveolar type II epithelial cells. J Pharm Sci 2017;106:2868-2872. 